\title{
Evaluation of the impact of high-tone therapy on cognitive disorders and quality of life in the complex treatment of patients with comorbid pathology
}

\author{
SHMAKOVA Iryna, PANINA Svitlana, MYKHAYLENKO Volodymyr \\ Odessa National Medical University, Odessa, Ukraine
}

Editor: Constantin Munteanu, Romanian Association of Balneology, office@bioclima.ro

Reviewers: Silisteanu Sinziana Calina and Rotariu Mariana

*Corresponding authors: Volodymyr Mykhaylenko, E-mail: vlmykhaylenko@gmail.com

\begin{abstract}
Introduction. Comorbidity is an independent risk factor for mortality and significantly influences the prognosis and quality of life.

Purpose: to evaluate the impact of high-tone HiTOP 4 touch therapy on cognitive disorders and quality of life in the complex treatment of patients with comorbid pathology.

Methods: complex treatment of 2 groups of patients with inclusion in the basic treatment regimen of high-tone therapy was carried out - a total of 80 patients (men - 34, women - 46) aged 41 to 79 years old, group I - patients with hypertension and chronic cerebral ischemia (CCI) - 38 patients and group II - patients with hypertension, CCI and concomitant diabetes mellitus (DM) type 2 - 42 patients. The average age in group I was 61.5, in group II - 65.5. Group I received lisinopril and amlodipine in one tablet, group II received metformin in addition to the above therapy. Both groups received a course of 10 sessions of hightone therapy using the device HiTOP 4 touch (Germany) according to the general method: 2 electrodes on the feet, 2 on the forearms and one on the neck-collar area. All the patients were assessed for their cognitive condition, degree of anxiety and depression, and estimated for quality of life before and after a course of high-tone therapy. In order to do this, we used valid assessment tests, such as the Montreal Cognitive Assessment Scale (MoCA), the Hospital Anxiety and Depression Scale (HADS), and the SF-36 Quality of Life Questionnaire. Results: the course of high-tone therapy for patients with hypertension and CCI led to improved quality of life, on all indicators of the SF-36 scale, except for pain intensity, increased cognitive functions by 3.52 points on the MoCA scale, reduced anxiety by 2.06 points and depression by 1.92 points on the HADS scale. The course of high-tone therapy for patients with CCI, hypertension and type 2 DM resulted in a significant improvement of 5 out of 8 quality of life indicators on the SF-36 scale, cognitive functions by 2.27 points on the MoCA scale and reduced anxiety by 4.3 points, and depression by 0.53 points on the HADS scale.

Conclusion: the inclusion of high-tone therapy in the complex treatment of patients with comorbid pathology improves cognitive functions, reduces anxiety and depression, improves quality of life.
\end{abstract}

Keywords: comorbid pathology, high-tone therapy, cognitive functions, anxiety, depression, quality of life,

\section{INTRODUCTION}

Comorbidity is an independent risk factor for mortality and significantly influences the prognosis of disease and life. The number of comorbid diseases increases with age: $10 \%$ - under the age of 19 and $80 \%$ - persons aged 80 and older [1].

According to a British study (2000-2014), the most common comorbidities in age- and sex-standardized models were hypertension (28.9\%), depression (23.0\%), arthritis (20.9\%), and asthma (17.7\%) and anxiety $(15.0 \%)$. In the presence of comorbid pathology, the severity of its course increases and the prognosis worsens; difficult primary diagnosis of the disease; treatment of several diseases requires taking into account the interaction of drugs; a significant increase in medical resources is needed; risks of side effects increases; patients' adherence to treatment decreases; the need for additional research grows [2].

The prevalence of dementia reaches 40 million people worldwide and is expected to double every 20 years to more than 110 million in 2050. Vascular dementia is the second most common disease after Alzheimer's disease, which is estimated to account for about $20 \%$ of cases [3, $4,5]$. Type 2 DM doubles the risk of dementia and Alzheimer's disease. It has been suggested that Alzheimer's disease may be a consequence of a particular form of cerebral insulin resistance and impaired glucose regulation $[6,7]$.

Patients with vascular dementia have a lower rate of decline in cognitive function in comparison with patients with Alzheimer's disease. The progression of vascular cognitive impairment can be slowed or stopped by using optimal approaches to therapy that emphasizes the need to consider new methods of physiotherapy, which include high-tone therapy [8].

Complex treatment corresponds to the WHO's direction "Rehabilitation 2030": in-depth integration of rehabilitation services into the health care system and strengthening of cross-sectoral linkages to meet the needs 
of population and the inclusion of rehabilitation services into the range of public health services. Currently, 2.4 billion people in the world have health problems that need rehabilitation [9].

High-tone therapy is a new step in the field of electrotherapy. Its main mechanism of action is a direct effect on cell metabolism, in order to transfer as much energy as possible through high frequencies in the range between 4096 and 32768 Hertz. Capacity frequencies up to $5000 \mathrm{~mW}$ enter directly into the body. The main direction of the action of high-tone therapy is to increase the number of mitochondria, which play an important role in comorbid diseases [10].

In order to date, the mitochondrial bioenergetic etiology of many diseases has been proven. The bioenergetic paradigm of metabolic and degenerative diseases, cancer and aging was proposed by Dr. Douglas Wallace and he was later awarded the Paul Jansen Prize by Johnson \& Johnson in the field of biomedical research in 2017. This bioenergetic paradigm is presented in Figure 1 [11].

The brain needs a lot of energy, especially in conditions of insufficient blood supply in the process of chronic cerebral ischemia. It is necessary to maintain the resting potential, to restore the concentration of ions after synaptic transmission (from 40 to $60 \%$ of ATP energy), to support synthetic and catabolic processes. The brain has neither glucose nor ATP reserves, so the brain is critically dependent on the every second supply of glucose, oxygen and ATP production, which occurs mainly in mitochondria [12].

Purpose: to evaluate the impact of high-tone HiTOP 4 touch therapy on cognitive disorders and quality of life in the complex treatment of patients with comorbid pathology.

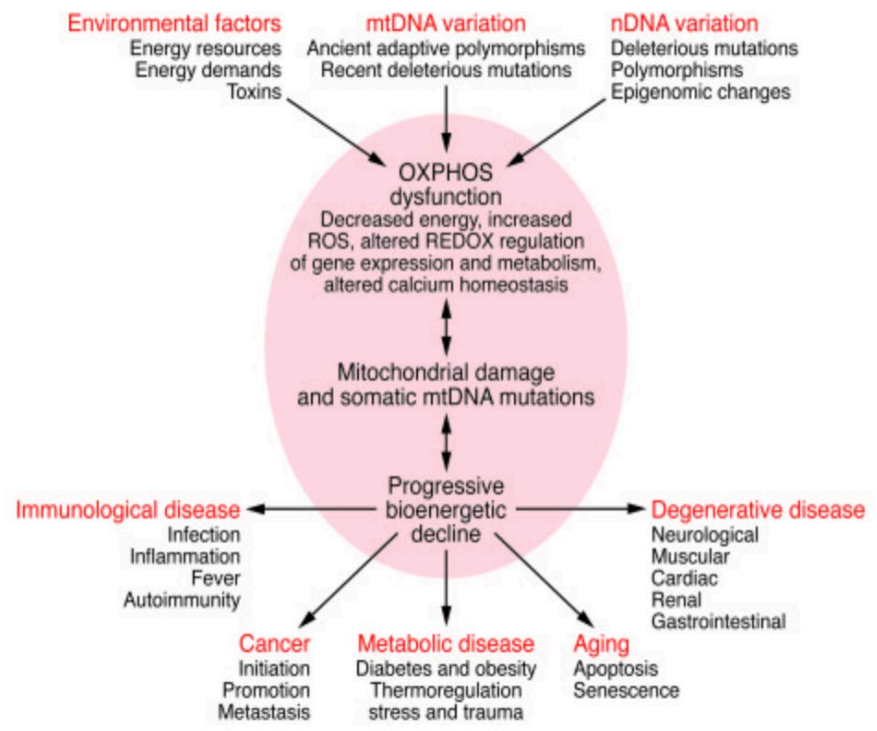

Fig.1 Bioenergetic paradigm for metabolic and degenerative diseases, cancer, and aging.
Materials and methods. Complex treatment of 2 groups of patients with inclusion in the basic treatment regimen of high-tone therapy - a total of 80 patients (men - 34, women - 46) aged 41 to 79 , group I - patients with hypertension and CCI - 38 patients and II group - patients with hypertension, CCI and concomitant type 2 DM - 42 patients. The average age in group I was 61.5 , in group II - 65.5. The diagnosis of hypertension was established based on the criteria of the recommendations of the European Society of Cardiology and the European Society of Hypertension (ESC/ ESH 2018) [13], the diagnosis of type $2 \mathrm{DM}$ was established according to the criteria of the American Diabetes Association (ADA 2018) [14]. The diagnosis of CCI, which corresponds to the term cerebral small vessel disease, was confirmed on the basis of the criteria of the recommendations of the European Stroke Organization (ESO 2018) [15]. Group I received lisinopril and amlodipine in one tablet, group II received metformin in addition to the above therapy. Both groups received a course of 10 sessions of high-tone therapy using the device HiTOP 4 touch (Germany) according to the general method: 2 electrodes are placed on the feet, 2 - on the forearms and one - on the neckcollar area.

All the patients were assessed for the level of their cognitive condition, degree of anxiety and depression, and estimated for quality of life before and after a course of high-tone therapy. In order to do this, we used valid assessment tests, such as the Montreal Cognitive Assessment Scale (MoCA), the Hospital Anxiety and Depression Scale (HADS), and the SF-36 Quality of Life Questionnaire.

Among the screening scales for revealing cognitive disorders, the most common are the Montreal Cognitive Assessment Scale (MoCA) and the Mini Mental State Examination (MMSE). The MoCA scale is considered to be more sensitive to vascular cognitive impairments than the MMSE scale [16].

The sensitivity of the MoCA scale to mild cognitive impairments is $90 \%$, and to Alzheimer's disease is $100 \%$. MoCA is an "ideal" screening test for moderate cognitive impairments. The sensitivity of the MMSE scale to mild cognitive impairments is $18 \%$, and to Alzheimer's disease is $78 \%$. MMSE is more suitable for detecting dementia, with a low sensitivity to mild cognitive deficit [17].

Assessment of the quality of life of patients with DM and comorbidities is a necessary tool in the practice of physicians, which is confirmed by the global interest in this issue - over the past 5 years, 1950000 articles have been published [18].

SF-36 consists of 8 scales, where a higher index proves a higher quality of life:

1) Physical Functioning (PF), which reflects the degree to which physical condition limits the performance of 
physical activity. Low indexes on this scale indicate that the patient's physical activity is significantly limited by his health.

2) Role-functioning due to physical condition (RolePhysical Functioning - RP) - the impact of physical condition on daily role-playing activities. Low indexes on this scale indicate that daily activities are significantly limited by the patient's physical condition.

3) The intensity of pain (Bodily Pain - BP) and its impact on the ability to engage in daily activities, which also includes work at home and outside the home. Low indexes on this scale indicate that pain significantly limits the patient's activity.

4) General Health (GH) - patient's assessment of his current health and treatment prospects. The lower the index on this scale, the lower the health index.

5) Vital activity (Vitality - VT) means feeling full of strength and energy or, conversely, exhausted. Low indexes indicate fatigue and decreased vital activity of the patient.

6) Social Functioning (SF) is defined by the degree to which a physical or emotional condition limits social activity or communication. Low indexes confirm a significant restriction of social contacts, a decrease in the level of communication due to the deterioration of physical and emotional state.

7) Role-functioning due to emotional state (RoleEmotional - RE) involves assessing the degree to which the emotional state interferes with work or other daily activities (including large expenditures of time, reducing the amount of work, decreasing its quality). Low indexes on this scale are interpreted as a limitation in the performance of daily work due to deteriorating emotional state.

8) Mental Health (MH) characterizes mood, the presence of depression, anxiety, a general indicator of positive emotions. Low rates indicate the presence of depressive, anxious feelings, mental distress.

The calculation of all values for the assessment of quality of life was performed in accordance with the instructions for processing data obtained using the SF-36 questionnaire and computer programs, according to special algorithms $[19,20]$.

Statistical analysis of the obtained data was performed using the packages STATISTICA 7.0 and Microsoft EXCEL 2003 with the integration of AtteStat 12.5, SISA Internet calculator (Simple Interactive Statistical Analysis). The average sample values of quantitative characteristics are given in the form of $\mathrm{M} \pm \mathrm{m}$, where $\mathrm{M}$ is the sample average, $\mathrm{m}$ is the mean error. Shares (percentages) are presented with 95\% confidence intervals. In all the procedures of statistical analysis when testing null hypotheses, the critical level of significance $p$ was assumed to be equal to 0.05 .
Results and discussion. The results of MoCA testing before and after the rehabilitation course in group I (hypertension, CCI) are presented in Figure 2.

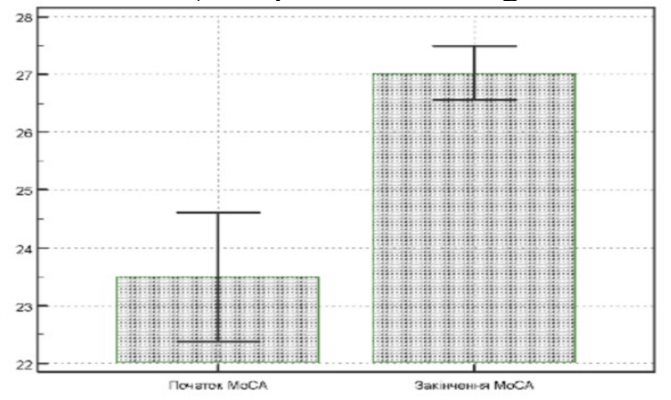

Fig. 2. Results of the MoCA test before and after undergoing high-tone therapy in group I.

After the course of high-tone therapy, the results of the MoCA test increased by 3.52 points: from 23.5 to 27.02 points $(\mathrm{p}<0.001)$.

Data from testing of cognitive disorders in patients of group II are presented in Figure 3.

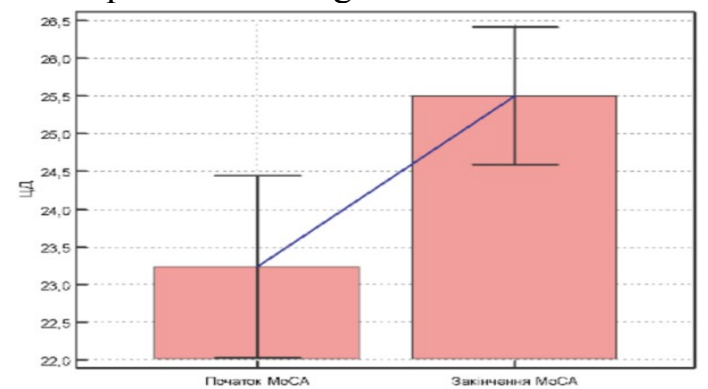

Fig. 3. Results of the MoCA test before and after undergoing high-tone therapy in group II.

The initial indicators of MoCA testing in group II were 23.23 points, and after the course with the use of hithtone therapy - 25.5 points. Improvement of cognitive functions by 2.27 points $(\mathrm{p}<0.05)$. High-tone therapy for patients of group II is less effective that fact can be explained by more complex metabolic brain damage in concomitant type 2 diabetes, namely impaired insulin signaling, neuroinflammatory and oxidative stress, as well as defects in mitochondrial metabolism and regulation of coactivator $1 \alpha$ receptor, sirtuin proliferator and peroxisome proliferator of SIRT-PGC-1 $\alpha$ receptor and tau protein signaling. Cognitive disorders can occur in the earliest stages of diabetes and is further exacerbated by metabolic syndrome. It should be noted that the strengthening of glycemic control in type 2 diabetes is not associated with improved cognitive function [21].

Cognitive deficits in CCI are manifested not only by slowing of mental activity, impaired attention, decreased speech activity and memory impairment, but also by affective and personality changes (depression, apathy, abulia, anxiety, irritability, emotional lability, 
deceleration) that in case of progressing can lead to the development of vascular dementia. Determining the level of anxiety and depression in this group of patients is extremely important, as in the presence of increased anxiety increases the likelihood of fatal myocardial infarction by 2.3 times, and the risk of sudden death increases by 4.5 times $[22,23]$. According to a study by the Helmholtz Center in Munich ( $\mathrm{n}=3428$ men, 45-74 years, 10 years of follow-up), it was determined that the effects of depression on the cardiovascular system have the same effect as obesity and high cholesterol. Therefore, it has been recommended to standardize diagnostic tests to check for depression in patients with heart disease, as it plays a vital role in the impact on their health [24].

The level of anxiety and depression using the Hospital Anxiety and Depression Scale (HADS) before and after a course of high-tone therapy in group I is presented in Figure 4.

In group I (hypertension, CCI) the level of anxiety at the beginning was 8.63 points, which corresponds to subclinically expressed anxiety, and after undergoing a course of high-tone therapy -6.57 points, i.e. the level of anxiety decreased by 2.06 points $(\mathrm{p}<0.01)$.

Before treatment, the level of depression was at the upper limit of normal - 6.86 points, and then decreased to normal -4.94 points $(-1.92$ points $),(\mathrm{p}<0.05)$.

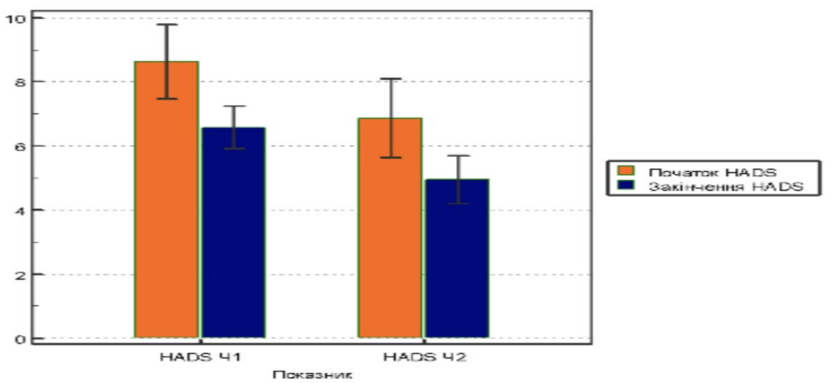

Fig. 4. The level of anxiety and depression on the HADS scale before and after the course of high-tone therapy in group I.

In determining the level of anxiety and depression in group II the results were obtained and presented in Figure 5.
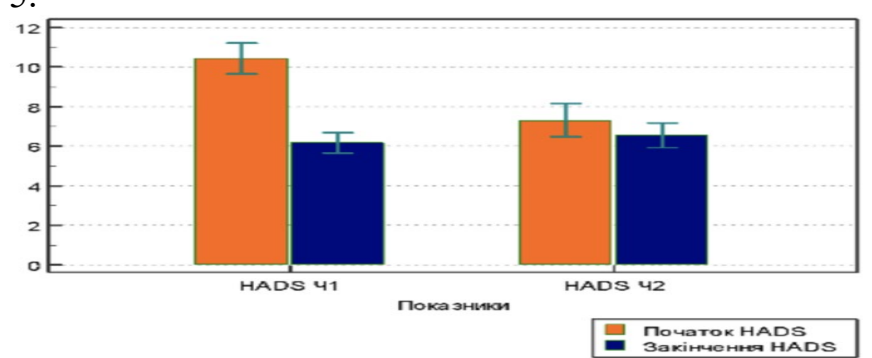

Fig.5. Level of anxiety and depression on the HADS scale before and after the course of high-tone therapy in group II
In group II, the initial level of anxiety was 1.77 points higher than in group I, and amounted to 10.4 points, which corresponds to clinically pronounced anxiety. After undergoing a course of high-tone therapy it was 6.1 points, i.e. returned to normal, the difference was 4.3 points $(\mathrm{p}<0.01)$.

The level of depression at the beginning of therapy was 7.03 points, which corresponds to subclinically expressed depression, and after undergoing a course of high-tone therapy, it decreased by 0.53 points to normal ( 6.5 points $)$ $(p<0.05)$.

A comparative analysis of the initial data of groups I and II for each criterion for assessing the quality of life of SF36 was carried out. It is presented in table 1 and figure 6 .

Table 1 - Comparison of the original data of groups I and II on the scale SF-36

\begin{tabular}{|c|c|c|c|}
\hline $\begin{array}{c}\text { Quality of life } \\
\text { indicators }\end{array}$ & $\begin{array}{c}\text { I group } \\
\text { (hypertension, } \\
\text { CCI) } \pm \mathrm{m}\end{array}$ & $\begin{array}{c}\text { II group } \\
\text { (DM, } \\
\text { hypertension, } \\
\text { CCI) } \pm \mathrm{m}\end{array}$ & $\mathrm{p}$ \\
\hline $\mathrm{PF}$ & $76,84 \pm 2,85$ & $57,38 \pm 4,1$ & $<0,001$ \\
\hline $\mathrm{RP}$ & $50 \pm 4,78$ & $55,57 \pm 4,51$ & $>0,05$ \\
\hline $\mathrm{BP}$ & $58,89 \pm 4,31$ & $43,19 \pm 3,79$ & $<0,05$ \\
\hline $\mathrm{GH}$ & $62,23 \pm 2,37$ & $49,38 \pm 3,43$ & $<0,01$ \\
\hline $\mathrm{VT}$ & $47,5 \pm 3,17$ & $41,9 \pm 3,87$ & $>0,05$ \\
\hline $\mathrm{SF}$ & $62,18 \pm 3,9$ & $60,83 \pm 3,85$ & $>0,05$ \\
\hline $\mathrm{RE}$ & $44,57 \pm 5,64$ & $41,33 \pm 6,55$ & $>0,05$ \\
\hline $\mathrm{MH}$ & $52,52 \pm 3,14$ & $40,14 \pm 1,84$ & $<0,01$ \\
\hline & & & \\
\hline
\end{tabular}

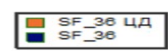

Fig. 6. Comparison of the original data on the SF-36 scale between groups I and II.

Note: BP - pain intensity, GH - general health, $\mathrm{MH}$ mental health, PF - physical functioning, RE - role functioning due to emotional state, RP - role functioning due to physical condition, SF - social functioning, VT vital activity.

According to the results of comparison of the initial data in group I (hypertension, CCI), the following indicators were higher: PF - physical functioning $(p<0.001)$, BP pain intensity $(\mathrm{p}<0.05)$, GH - general health $(\mathrm{p}<0.01)$, $\mathrm{MH}$ - mental health $(\mathrm{p}<0.01)$, which indicates a better initial general health of this group of patients than in patients with concomitant type $2 \mathrm{DM}$. Only one indicator was lower in group I compared to group II - RP - role functioning due to physical condition, but this indicator did not reach the level of statistical significance. 
It should be noted that according to the literature sources, patients with type $2 \mathrm{DM}$ have a low quality of life, which is often associated with depression, and also they have complications in the form of diabetic polyneuropathy, which also leads to a lower pain intensity and general condition indicators $[18,25]$.

The results of the assessment of quality of life before and after undergoing a course of high-tone HiTOP 4 touch therapy in group I (hypertension, CCI) are presented in Figure 7.

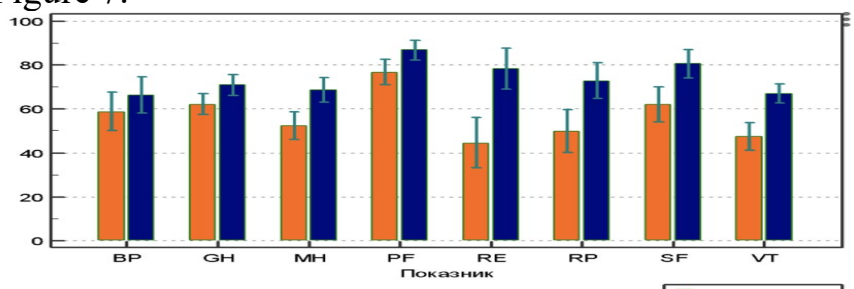

E Паиматок

Fig. 7. Indicators of the SF-36 scale in group I before and after undergoing high-tone therapy

In the pathogenesis of CCI in hypertension there is arteriosclerosis (lipohyalinosis) of small penetrating arteries and arterioles (hypertensive arteriopathy) [22]. The use of supra-tone frequency currents improves microcirculation, including in the brain, which can help restore the patient's psycho-neurological status and improve his quality of life. Thus, in group I, such indicators of the SF-36 scale as MH - mental health ( $\mathrm{p}$ $<0.001$ ), RE - role functioning due to emotional state ( $p$ $<0.001)$, VT - vital activity $(\mathrm{p}<0.001)$ greatly increased and reached the maximum statistical significance.

The use of high-tone therapy in group I led to an increase in quality of life in other indicators: GH - general health $(\mathrm{p}<0.05)$; PF - physical functioning $(\mathrm{p}<0,05)$; RP - role functioning due to physical condition $(\mathrm{p}<0.01)$, SF social functioning $(\mathrm{p}<0.01)$.

Only the indicator of BP - pain intensity did not reach the level of statistical significance ( $p>0.05)$. It should be noted that the intensity of pain in group I may have been due to concomitant pathology of the musculoskeletal system, which according to the literature occurs in $52 \%$ of patients with hypertension [26].

Figure 8 shows the dynamics of quality of life on the SF36 scale before and after undergoing a course of hightone therapy in group II.

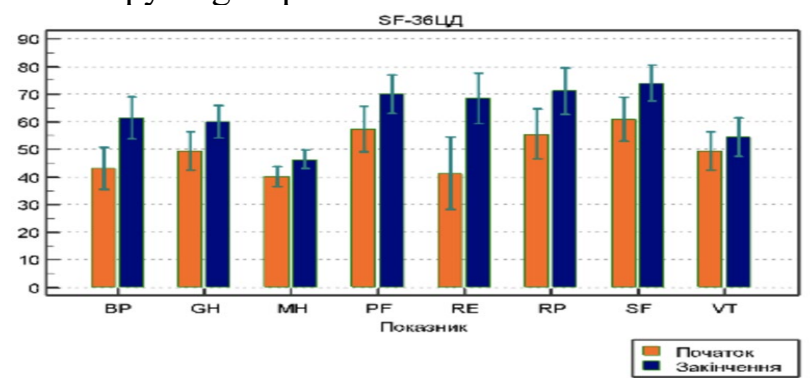

Fig. 8. Indicators of the SF-36 scale in group II before and after undergoing high-tone therapy
During the analysis of indicators after undergoing a course of high-tone therapy in group II, the following indicators of quality of life improved: BP - pain intensity ( $p<0,01)$, MH - mental health $(\mathrm{p}<0,05), \mathrm{RE}$ - role functioning due to emotional state $(\mathrm{p}<0.01)$, RP - role functioning due to physical condition $(\mathrm{p}<0.05)$, SF social functioning $(\mathrm{p}<0.05)$.

The indicator of pain intensity in group II of patients was mainly due to diabetic polyneuropathy as a complication of DM (according to ambulatory cards $80 \%$ of patients with type 2 diabetes). It indicated statistically significant regression after the rehabilitation course.

Thus, 5 out of the 8 indicators of quality of life on the SF-36 scale have undergone a statistically significant improvement, which proves the high efficiency of this rehabilitation method in comorbid pathology and substantiates its use. Up to this date, studies have shown little or moderate benefit in improving the quality of life of patients with DM [18].

Thus, the use of supra-tone frequency currents in patients with comorbid pathology (hypertension, CCI, type $2 \mathrm{DM}$ ) significantly improves cognitive function, reduces anxiety and depression, improves quality of life.

To understand the mechanism of action of supra-tone frequency currents on the pathogenesis of CCI, we must remember that we primarily influence the mitochondria. As mentioned above, the main mechanism of action of high-tone therapy is to increase energy balance due to influence on mitochondria. To date, mitochondrial function in lymphocytes has been shown to be a potential biomarker of neurodegeneration progression, as peripheral mitochondrial function is associated with moderate cognitive disorders due to impaired mitochondrial ATP production, oxidative stress, and increased proton outflow. Thus, high-tone therapy is pathogenetically substantiated in the cerebral small vessel disease [27].

High anxiety is often combined with several pathologies of the nervous system. Existing treatments (e.g., cognitive-behavioral therapy and anxiolytic drugs) help to achieve remission in only $50 \%$ of adults, while more than a third of patients with anxiety disorders are resistant to treatment. Due to the limited effectiveness of existing (psychological and pharmacological) treatments, there is a need to develop new methods of treatment based on a deeper understanding of the molecular mechanisms underlying the pathogenesis of anxiety $[28,29,30]$.

Recent studies show that impaired neuroplasticity and stability may underlie the pathophysiology of anxiety disorders. It has been suggested that the release of mitochondrial calcium $(\mathrm{Ca} 2+)$ plays a key role in modulating the tone of synaptic plasticity in various neuroanatomical areas, including those involved in the pathophysiology of anxiety. Mitochondria also play a role in calcium homeostasis. Calcium $(\mathrm{Ca} 2+)$ is the main 
secondary messenger that helps regulate both neurotransmission and short-term and long-term plasticity of neurons in the brain [31]. The fine spatial and temporal organization of intracellular calcium signals is important for the functioning of the central nervous system (CNS). Signals are transmitted throughout the CNS due to local changes in the concentration of $\mathrm{Ca} 2+$. Thus, $\mathrm{Ca} 2+$ signals that are required for synaptic transmission and, therefore, for the transmission of information throughout the CNS are transmitted to the mitochondria, where it is assumed that $\mathrm{Ca} 2+$ modulates mitochondrial metabolism with activation of the tricarboxylic acid cycle, ATP synthase and aspartate carrier, followed by ATP increase $[32,33]$.

A study of the relative role of mitochondrial buffering and the endoplasmic reticulum $\mathrm{Ca} 2+$ showed that dendritic mitochondria rapidly accumulate $\mathrm{Ca} 2+$, while the endoplasmic reticulum shows a more delayed increase in $\mathrm{Ca}^{2+}$ during high-frequency stimulation. Thus, it is possible that the regulation of mitochondrial function plays an important role in regulating the synaptic power of neurons that mediate complex behavior [34]

Identification of key pathophysiological components is vital for the development of new approaches to therapy, such as the use of high-tone therapy, which directly influences the mechanism of metabolic dysfunction of the brain and has a confirmed result according to MoCA testing.

There is another extremely important mechanism in the effect of high-tone HiTOP 4 touch therapy on mitochondria in the pathogenesis of many neurodegenerative diseases. N-acetylaspartate (NAA) is synthesized in the mitochondria of neurons from aspartate and acetyl-coenzyme by aspartate-Nacetyltransferase (ANAT), and is a precursor of an important neurotransmitter and one of the most common dipeptides contained in the human brain. That is, influencing the mitochondria, we enhance the synthesis of NAA in the neurons of the brain. After synthesis of neurons in the mitochondria, NAA enters glia cells oligodendrocytes and astrocytes. It is established that NAA is one of the main regulators of osmotic processes in the brain and is considered as a signaling molecule in the system of neuron-glia interaction, also involved in the synthesis of brain lipids, can serve as a source of aspartate, acyl groups in myelin synthesis. From neurons to oligodendrocytes, it enters as a key metabolite for myelin synthesis. Therefore, by influencing the mitochondria, we trigger neurorepair processes that are important in many diseases of the brain, including $\mathrm{CCI}$ [35].

Thus, the use of high-tone therapy in patients with comorbid pathology (hypertension, CCI, type 2DM) improves cognitive impairments, reduces anxiety and depression, improves quality of life, which is probably due to the influence of supra-tone frequency currents on the molecular mechanisms of cells, and it is on the mitochondria.

\section{Conclusions}

1. Patients with hypertension and CCI indicators on the scale of quality of life SF-36 had significantly better indicators of physical functioning $(\mathrm{p}<0.001)$, pain intensity $(\mathrm{p}<0.05)$, general health $(\mathrm{p}<0.01)$, mental health $(\mathrm{p}<0.01)$ in comparison with patients with concomitant DM. The level of anxiety was significantly higher in the group patients with type 2 DM than in the other group of patients with hypertension $(\mathrm{p}<0.05)$.

2 . The course of high-tone therapy given to patients with hypertension and CCI led to an improved quality of life, on all indicators of the SF-36 scale, except for pain intensity $(p>0.05)$, increased cognitive function by 3.52 points on the MoCA scale 0.001 ), reduction of anxiety by 2.06 points $(\mathrm{p}<0.01)$ and depression by 1.92 points $(\mathrm{p}$ $<0.05)$ on the HADS scale.

3. Carrying out a course of high-tone therapy in patients with CCI, hypertension and type 2 DM led to a significant improvement of 5 out of 8 indicators of quality of life on the SF-36 scale, cognitive functions by 2.27 points $(p<0.05)$ on the MoCA scale, reduction of anxiety by 4.3 points $(\mathrm{p}<0.01)$ and depression by 0.53 points $(\mathrm{p}<0.05)$ on the HADS scale.

\section{References}

1. Oganov RG, Denisov IN, Simanenkov VI, Bakulin IG, Bakulina NV, Boldueva SA, et al. Comorbid pathology in clinical practice. Clinical guidelines. Cardiovascular therapy and prevention [Internet]. 2017 [cited 2021 Okt. 12];16(6):5-56. Available at: https://doi.org/10.15829/17288800-2017-6-5-56 [In Russian]

2. Tran J, Norton R, Conrad N, Rahimian F, Canoy D, Nazarzadeh $\mathrm{M}$, et al. Patterns and temporal trends of comorbidity among adults patients with incident cardiovascular disease in the UK between 2000 and 2014: A population-based cohort study. PLoS Med. 2018 Mar;15(3):e1002513. doi: 10.1371/journal.pmed.1002513

3. Nasonova TI, Klimenko OV, Kolosova TV, Gorieva GV, Golovchenko Yu.I, Tishkevich OV, et al. Neurovegetative and cognitive impairments, associated with anxiety in patients of the middle age caused by cerebrovascular pathology. Semeynaia meditsina. 2017;2:97-102. [In Ukrainian]

4. Prince M, Bryce R, Albanese E, Wimo A, Ribeiro W, Ferri CP. The global prevalence of dementia: a systematic review and metaanalysis. Alzheimers Dement. 2013;9(1): 63-75.

5. Rizzi L, Rosset I, Roriz-Cruz M. Global epidemiology of dementia: Alzheimer's and vascular types. Biomed Res Int. 2014;2014:908915.doi: 10.1155/2014/908915

6. de la Monte SM. Brain insulin resistance and deficiency as therapeutic targets in Alzheimer's disease. Curr Alzheimer Res. 2012;9(1):35-66.

7. Akter K, Lanza EA, Martin SA, Myronyuk N, Rua M, Raffa RB. Diabetes mellitus and Alzheimer's disease: 
shared pathology and treatment? $\mathrm{Br} \mathrm{J}$ Clin Pharmacol. 2011;71(3):365-76. doi: 10.1111/j.1365-2125.2010.03830.x

8. Farooq MU, Min J, Goshgarian C, Gorelick PB. Pharmacotherapy for Vascular Cognitive Impairment. CNS Drugs. 2017;31(9):759-76. doi: 10.1007/s40263-017-0459-3

9. World Health Organization. Available at: https://www.who.int/ru/news-room/factsheets/detail/rehabilitation

10. HiToP ${ }^{\circledR}$ high-tone therapy according to Dr. May [Internet]. Available at: https://www.dr-med-may.de/hitophochtontherapie

11. Wallace DC. A mitochondrial bioenergetic etiology of disease. J Clin Invest. 2013 Apr 1;123(4):1405-1412. doi: 10.1172/JCI61398

12. Mergenthaler P, Lindauer U, Dienel GA. Sugar for the brain: the role of glucose in physiological and pathological brain function.Trends Neurosci. 2013 Oct;36(10):587-97. doi: 10.1016/j.tins.2013.07.001

13. 2018 ESC/ESH Clinical Practice Guidelines for the Management of Arterial Hypertension [Internet]. Available at: https://www.escardio.org/Guidelines/Clinical-PracticeGuidelines/Arterial-Hypertension-Management-of

14. Standards of Medical Care in Diabetes - 2018 Abridged for Primary Care Providers. Clinical Diabetes [Internet]. 2018 [cited 2021 Oct 12];36(1):14-37. Available at: https://doi.org/10.2337/CD17-0119

15. Wardlaw JM, Debette S, Jokinen H, De Leeuw FE, Pantoni L, Chabriat $\mathrm{H}$, et al. ESO Guideline on covert cerebral small vessel disease. European Stroke Journal. 2018;6(2):CXI-CLXII. doi: 10.1177/23969873211012132

16. Clinical protocol of medical assistance ischemic stroke (emergency, first, second (special) medical assistance, medical rehabilitation): Order of the Ministry of Health No. 602 dated 03.08.2012. [In Ukrainian]

17. Nasreddine ZS, Phillips NA, Bédirian V, Charbonneau S, Whitehead V, Collin I, et al. The Montreal Cognitive Assessment, MoCA: a brief screening tool for mild cognitive impairment. J Am Geriatr Soc. 2005;53(4):695-9. doi: 10.1111/j.1532-5415.2005.53221.x.

18. Trikkalinou A, Papazafiropoulou AK, Melidonis A. Type 2 diabetes and quality of life. World J Diabetes. 2017 Apr 15;8(4):120-9. doi: 10.4239/wjd.v8.i4.120

19. Belialova NS. Instructions for processing data obtained using the SF-36 questionnaire [Internet]. [cited 2021 Oct 12]. Available at: https://therapy.irkutsk.ru/doc/sf36a.pdf [In Russian]

20. The Health Institute (THI). International Resource Center (IRC) for Health Care Assessment. Scoring exercise for the MOS SF-36 Health Survey. Boston;1992.

21. Zilliox LA, Chadrasekaran K, Kwan JY, Russell JW. Diabetes and Cognitive Impairment. Current Diabetes Reports. 2016;16(9):87. doi: 10.1007/s11892-016-0775-x

22. Antipenko EA, Gustov AV. Chronic cerebral ischemia: current state of the problem. Meditsinskiy sovet. 2016;(19):38-43. [In Russian]

23. Kessler RC, Chiu WT, Demler O, Merikangas KR, Walters EE. Prevalence, severity and comorbidity of 12-month DSM-IV disorders in the National Comorbidity Survey
Replication. Arch Gen Psychiatry. 2005;62(6):617-27. doi: 10.1001/archpsyc.62.6.617.

24. Ladwig K-H, Baumert J, Marten-Mittag B. Room for depressed and exhausted mood as a risk predictor for allcause and cardiovascular mortality beyond the contribution of the classical somatic risk factors in men. Atherosclerosis. 2017;(257):224-31.

doi: 10.1016/j.atherosclerosis.2016.12.003.

25. Douglas W Zochodne. The challenges of diabetic polyneuropathy: a brief update. Curr Opin Neurol. 2019;32(5):666-75.

doi: 10.1097/WCO.0000000000000723.

26. Lukianchuk E. Comorbidity in osteoarthroshis: a rational approach to the choice of a non-steroidal anti-inflammatory drug. Ukrainskyi Revmatologichnyi Zhurnal [Internet]. 2014 [cited 2021 Oct 12];58(4). Available from: https://www.rheumatology.kiev.ua/article/7498/komorbidn ost-pri-osteoartroze-racionalnyj-podxod-k-vyborunesteroidnogo-protivovospalitelnogo-preparata [in Russian]

27. Apaijai N, Sriwichaiin S, Phrommintikul A, Jaiwongkam T, Kerdphoo S, Chansirikarnjana S, et al. Cognitive impairment is associated with mitochondrial dysfunction in peripheral blood mononuclear cells of elderly population. Sci Rep. 2020;10(1):21400. doi: 10.1038/s41598-02078551-4.

28. Olfson M, Mojtabai R, Merikangas K, Compton WM, Wang S, Grant BF. et al. Reexamining associations between mania, depression, anxiety and substance use disorders: results from a prospective national cohort. Mol Psychiatry [Internet]. 2017 [cited 2021 Oct 12];(22):23541. Available from: https://doi.org/10.1038/mp.2016.64

29. Bystritsky, A. Treatment-resistant anxiety disorders. Mol Psychiatry [Internet]. 2006 [cited 2021 Oct 12];(11):80514. Available from: https://doi.org/10.1038/sj.mp.4001852

30. Calhoon G, Tye K. Resolving the neural circuits of anxiety. Nat Neurosci [Internet]. 2015 [cited 2021 Oct 12];(18):1394-404. Available from: https://doi.org/10.1038/nn.4101

31. Schweitzer N. Pegging pathology on mitochondrial dysfunction. Scientist. 2004;18:28.

32. Duchen MR. Mitochondria, calcium-dependent neuronal death and neurodegenerative disease. Pflugers Arch. 2012;464:111-21.

33. Pardo B, Contreras L, Serrano A, Ramos M, Kobayashi K, Iijima $\mathrm{M}$, et al. Essential role of aralar in the transduction of small $\mathrm{Ca} 2+$ signals to neuronal mitochondria. J Biol Chem. 2006;281:1039-47.

34. Pivovarova NB, Pozzo-Miller LD, Hongpaisan J, Andrews SB. Correlated calcium uptake and release by mitochondria and endoplasmic reticulum of CA3 hippocampal dendrites after afferent synaptic stimulation. $\mathrm{J}$ Neurosci. 2002;22:10653-61.

35. Baymeeva NV, Miroshnichenko II. N-acetylaspartate is a biomarker of mental and neurological disorders. Zhurnal Nevrologii i Psikhiatrii im. S.S. Korsakov [Internet]. 2015 [cited Oct 2021. 12];115(8):94-8. Available at: https://doi.org/10.17116/jnevro20151158194-98 [In Russian] 